\title{
Kernos
}

Revue internationale et pluridisciplinaire de religion grecque antique

30 | 2017

Varia

\section{Il soldato e l'atleta. Guerra e sport nella Grecia antica}

\section{Vincent Cuche}

\section{(2) OpenEdition \\ Journals}

Édition électronique

URL : http://journals.openedition.org/kernos/2546

DOI : 10.4000/kernos.2546

ISSN : 2034-7871

\section{Éditeur}

Centre international d'étude de la religion grecque antique

\section{Édition imprimée}

Date de publication : 1 octobre 2017

Pagination : 356-359

ISSN : 0776-3824

\section{Référence électronique}

Vincent Cuche, « II soldato e l'atleta. Guerra e sport nella Grecia antica », Kernos [En ligne], 30 | 2017,

mis en ligne le 01 octobre 2017, consulté le 24 septembre 2020. URL : http://journals.openedition.org/ kernos/2546 ; DOI : https://doi.org/10.4000/kernos.2546

Ce document a été généré automatiquement le 24 septembre 2020.

Kernos 


\title{
Il soldato e l'atleta. Guerra e sport nella Grecia antica
}

\author{
Vincent Cuche
}

\section{RÉFÉRENCE}

Paola ANGELI BERNARDINI, Il soldato e l'atleta. Guerra e sport nella Grecia antica, Bologne, Il

Mulino, 2016, 1 vol. 13,5 × 21 cm, 270 p. (Saggi, 845). ISBN : 978-88-15-26525-8.

1 On connaît l'erreur d'interprétation du devin Teisamenos : ayant appris d'un oracle delphique qu'il remporterait cinq agônes, il s'était essayé au pentathlon et avait connu l'échec à Olympie. Il ne resta pas longtemps victime de l'ambiguïté de Loxias, puisque les Spartiates, plus habiles que lui, devinèrent que le terme agôn désignait ici une bataille rangée et remportèrent cinq grandes victoires grâce à son art mantique (Hdt., IX, 33-35).

2 C'est cette idée d'une équivalence possible entre l'agôn sportif et l'agôn militaire qui est au cœur de l'ouvrage de Paola Angeli Bernardini. L'A. entend en effet mener une comparaison systématique entre la figure du soldat et celle du guerrier, du viII ${ }^{\mathrm{e}}$ au $\mathrm{v}^{\mathrm{e}}$ siècle avant notre ère, période définie comme un âge d'or des concours sportifs. Le parcours se fait sur cinq chapitres, durant lesquels il s'agit d'étudier les éléments qui rapprochent ou qui différencient ces deux types d'expérience agonale, principalement $\mathrm{au}$ niveau de leur matrice idéologique commune, mais également dans leurs composantes religieuses, civiques et littéraires. L'entreprise se veut ambitieuse, tout en restant dans les limites d'un essai accessible au grand public, comme en témoignent l'appareil des notes très réduit et la présence d'indices où les termes techniques grecs sont traduits en italien.

3 Dès le premier chapitre, l'A. s'inscrit en faux contre tout un pan de la recherche actuelle, qui tend plutôt à minimiser, voire à nier, le caractère agonistique de la guerre grecque $^{1}$. Si le guerrier peut être rapproché de l'athlète, c'est que l'un et l'autre sont imprégnés d'une même pulsion idéologique, la «mentalità agonale». Cette forme 
particulière d'esprit de compétition, qui se manifeste d'ailleurs dans bien d'autres domaines de la société grecque, trouve sa définition quintessentielle dans l'exhortation

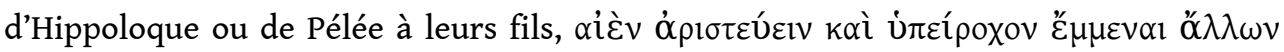
(Iliade VI, $208 ; \mathrm{XI}, 784$ ). Il est vrai que, dans le cadre homérique, le passage du combat à la compétition se fait aisément puisque les acteurs sont les mêmes et qu'ils y apportent la même fougue et la même ardeur à l'emporter ; le duel organisé, tel celui entre Hector et Ajax, fournissant un moyen terme aux deux expériences. En ce qui concerne les époques historiques, les choses sont évidemment plus complexes, et l'A. s'emploie à recenser les points d'accord et de désaccord entre pratique guerrière et pratique sportive. Parmi les différences, la volonté homicide et la férocité guerrières (encore que ce dernier point pourrait être nuancé, si l'on songe à la violence du pugilat et du pancrace), ainsi que l'aspect collectif de la guerre hoplitique, opposé à l'individualisme du sport antique; tandis que la présence de public et d'arbitres est propre à la compétition. Du côté des ressemblances, l'usage d'un vocabulaire commun (ponos, kindunos), le respect de règles et de limites, la quête de la gloire, la valorisation du corps masculin, forgé dans le gymnase et statufié dans l'espace public. Dans le sens des affinités également, l'A. cite de nombreux dieux qui sont liés à l'un et l'autre domaines, à commencer par les figures centrales de Zeus et de Niké. Le raisonnement paraît parfois un peu trop abrégé: que Déméter (par exemple) soit honorée par une compétition lors des Éleusinia ne nous dit pas grand-chose du lien qu'elle entretient avec l'activité athlétique; plus généralement, le fait que diverses divinités interviennent dans deux domaines d'actions différents ne signifie pas automatiquement que les domaines en questions soient étroitement apparentés. Enfin, ce chapitre se focalise brièvement sur deux catégories sportives, les épreuves de course et les épreuves équestres, pour examiner leur rapport à la guerre; il est un peu paradoxal que les épreuves de combat (boxe, lutte, pancrace) n'aient pas droit à un pareil traitement.

Le chapitre suivant porte sur le moment conclusif de l'agôn sportif et guerrier: la victoire, son annonce et sa célébration. Après quelques remarques sur les processus de communication de l'information, l'A. expose les différentes étapes par lesquelles le guerrier (ou l'armée guerrière) et l'athlète victorieux acquièrent ce kleos qui constituait la motivation principale de leur combat. Dans l'un et l'autre cas, l'honneur et le prestige qui entourent le vainqueur se manifestent dès la fin du combat, par des péans ou de brefs chants de victoire, par des prix et des couronnes, avant d'être développés dans de plus larges compositions poétiques, pérennisés par des monuments ou commémorés par des inscriptions funéraires. Il n'est d'ailleurs pas rare dans ce genre de textes que les mérites militaires et sportifs du laudandus soient juxtaposés. Tout ceci fournit le cadre de formation d'une "verità agonale ", notion qui donne lieu à une belle réflexion de l'A., dans la droite ligne des Maitres de vérité de Marcel Detienne. La victoire étant ce qui affirme la valeur du vainqueur et ce qui l'inscrit dans une mémoire, l'agôn proclame donc une vérité (aletheia), qui s'oppose à l'oubli (lethé) auquel est condamné le vaincu; de ce point de vue, Olympie est le lieu de l'aletheia par excellence (Pind., ol. 8, 1-2). L'A retourne ensuite sur la question des nomoi de la guerre et du sport, dont le respect serait plus ancien que la formalisation.

5 Après une présentation des sources poétiques (chapitre 3), où figurent notamment quelques remarques intéressantes sur l'importance comparée de la thématique guerrière dans les poésies d'Alcée et d'Archiloque, l'A. en vient, dans son quatrième chapitre, au cœur du sujet, l'analyse des valeurs communes qui constituent l'identité du 
guerrier et de l'athlète. On y reparle du corps de ces derniers, idéalisé, statufié, subissant parfois l'aphanismos, ce qui entraîne alors la création d'un culte héroïque; souvent, le corps du défunt continue d'exercer sa puissance en servant de talisman à sa cité. On ne peut accepter en revanche l'affirmation de l'A. selon laquelle aucune cité n'accepte le corps d'un ennemi : non seulement les realia funéraires fournissent des contre-exemples - qu'on songe à la sépulture des Lacédémoniens au Céramique - mais le mythe même d'Eurysthée prouve qu'il est possible, pour une communauté, de réutiliser pour son propre compte la puissance talismanique d'un adversaire. Après les valeurs physiques viennent les vertus morales qui définissent l'arété sportive et militaire: andreia, karteria, philoponia, bref tout un système éthique, développé notamment par Pindare et Bacchylide à l'intention de l'aristocratie, mais adopté aussi par la mentalité civique. La clef de voûte de cette idéologie agonistique est incarnée par la figure d'Héraclès, dont la structure mythologique unit les aspects athlétiques et militaires. Par sa vie violente, faite de ponoi et de combats, le héros fournit un modèle unitaire à des personnages tels que Milon de Crotone ou Théogène de Thasos (dans le chapitre suivant, l'A. suggère qu'Atalante pouvait proposer, au moins sur le plan théorique, un équivalent féminin au modèle héracléen). D'autres points de contact entre sportifs et soldats - l'usage de l'aulos, le bouclier des concours argiens, l'hoplitodromie - sont également passés en revue.

6 C'est à la fin de ce quatrième chapitre que l'A. aborde la quaestio vexata du rapport entre mentalité agonistique et guerre hoplitique, en reprenant les exemples classiques de résolution du conflit par combat règlementé (guerre lélantine, duels de champions) ; la réflexion se poursuit dans le dernier chapitre, consacré à la place du soldat et de l'athlète dans la cité, où l'A. considère que la logique individuelle de l'agôn pouvait s'appliquer, sinon au soldat, du moins au stratège, voire à l'armée considérée comme une unité. Cette mentalité ne disparaît pas, bien au contraire, avec la professionnalisation des athlètes et des soldats (que l'auteur fait intervenir de manière contestable à partir du $\mathrm{IV}^{\mathrm{e}}$ siècle). Est également abordé, quoique brièvement, le problème, débattu dès l'Antiquité, de l'utilité militaire d'une formation athlétique. Enfin l'abondance des offrandes militaires à olympie, la création de concours commémorant des victoires militaires ou des stratèges offrent d'autres modes de contact entre guerre et sport. Sur ce dernier sujet, plutôt que les concours de Chersonèse en l'honneur de Miltiade l'Ancien, dont Hérodote (VI, 38) dit explicitement qu'ils sont liés à sa qualité d'oikiste, et non à ses succès sportifs ou militaires, je citerais plus volontiers l'agôn epitaphios que les Athéniens instituèrent probablement à la suite des guerres médiques (cf. IG I ${ }^{3}$ 523-525). Ce qui est indéniable, c'est que ces concours commémoratifs contribuaient à définir des lieux de mémoire perpétuant le souvenir des grandes victoires civiques et des grands hommes, l'arété mise en œuvre par les athlètes répondant à celle des exploits passés. Comme le souligne l'A., l'ensemble de ces concours, généralement façonnés suivant le modèle des compétitions de la Période, configurait un réseau des communautés grecques où politique et diplomatie n'étaient jamais loin du sport. L'ouvrage se conclut sur quelques réflexions plus générales sur la mystique de la victoire et son rapport au pouvoir, d'où le goût des monarques et des tyrans pour les concours athlétiques, que ce soient ceux de la Grèce antique ou ceux de notre époque contemporaine.

7 En somme, il s'agit d'un ouvrage à la matière fort riche, fourmillant de détails et de pistes de réflexion - peut-être un peu trop. Le lecteur risque d'être un peu déconcerté par la tendance de l'A. à multiplier les approches, à prendre des libertés avec le plan 
qu'elle s'était fixé et à effectuer des redites et des digressions. De manière plus problématique, certaines parties ne semblent guère participer du «confronto sistematico » entre sport et guerre annoncé par l'A. : le long chapitre 3 qui fait le tour des genres poétiques grecs paraît souvent déconnecté du sujet, et l'on ne comprend pas pourquoi le récit de la bataille de Salamine vient s'insérer dans la ligne argumentative du chapitre 4. Tout cela donne parfois l'impression que l'A. juxtapose, plus qu'elle ne les confronte, des réflexions sur la guerre antique et d'autres sur le sport; on regrette ainsi que les deux facettes d'Héraclès, le guerrier et l'athlète, soient traitées l'une après l'autre alors que le raisonnement même de l'A. incitait à chercher dans l'Alcide ce qui les unissait.

8 Quant à la thèse principale de cet ouvrage, à savoir l'unité et l'importance de la mentalité agonistique dans les domaines militaire et athlétique, elle ne peut qu'entraîner mon adhésion, mais il est dommage que l'A., sans doute par scrupule de répéter ses écrits antérieurs ${ }^{2}$, n'ait pas choisi de se confronter d'emblée à ce qu'elle a de problématique, y compris pour les Grecs antiques. Après tout, des élégies de Tyrtée à l' Anacharsis de Lucien, la parenté entre guerre et sport a fait l'objet de virulentes controverses, qui ne semblent pas avoir reçu de réponse définitive. L'athlète fait-il un bon soldat? Le soldat peut-il se targuer d'exploits individuels et ses mérites valent-ils ceux de l'athlète ? Il y a différentes manières de répondre à ces questions; l'une d'entre elles consiste, par exemple, à mettre en scène un duel entre un champion athlétique et un soldat: c'est ainsi que l'hoplite athénien Sophanès est loué pour avoir tué un champion argien du pentathlon (Hdt., VI, 92) ; inversement, Dioxippos, dans sa nudité athlétique, remporte la victoire sur un soldat macédonien tout armé (Diod. Sic., XVII, 100). On peut encore entrer dans le détail des épreuves et débattre de l'utilité de la boxe et de la lutte sur un champ de bataille, comme le fait Plutarque dans ses Propos de table (II, 5) ou Lucien dans l'Anacharsis. L'étude de ces discours ne peut faire l'économie d'une confrontation avec les réalités de la guerre antique, qui font elles-mêmes l'objet d'une constante réévaluation (par exemple, en ce qui concerne l'othismos) ${ }^{3}$. Selon la conception qu'on se fait de l'hoplitisme, les conclusions différeront. Qu'on insiste sur le caractère compact de la phalange et la méfiance vis-à-vis des exploits guerriers individuels, et l'activité athlétique apparaît souvent en dissonance avec l'activité militaire; de ce décalage de l'idéologie agonistique par rapport à la pratique, on a rendu compte de plusieurs manières. Qu'on adhère au contraire aux récentes théories en faveur d'une autonomie plus importante de l'hoplite au sein de la phalange, ou qu'on souligne le rôle formateur des gymnases, par exemple dans la montée en puissance de l'armée thébaine, et les deux formes d'expérience retrouvent une certaine parentét. Ce sont là des questions complexes, qui auraient sans doute mérité un plus long approfondissement.

9 Pour autant, ce livre est en soi une mine de réflexions stimulantes et nous rappelle à bon escient que tout comme la guerre, la pratique sportive s'accompagne d'un système idéologique qu'il convient d'analyser. Enfin, l'ouvrage est agréablement édité (malgré ses quelques coquilles, notamment dans les références anciennes et modernes ${ }^{5}$ ) et utilement accompagné d'une douzaine de figures en noir et blanc. 


\section{NOTES}

1. L'A. renvoie essentiellement à l'ouvrage de J. DAYTON, The Athletes of War: An Evaluation of the Agonistic Elements in Greek Warfare, Toronto, 2006, auquel on pourrait rajouter les articles de P. KRENTZ, « Deception in Archaic and Classical Greek Warfare », in H. VAN WEES (éd.), War and Violence in Ancient Greece, Londres, 2000, p. 167-200, et «Fighting By the Rules: The Invention of the Hoplite Agon », Hesperia 71 (2002), p. 21-39, ainsi que la synthèse de L. RAWLINGS, The Ancient Greeks at War, Manchester, 2007.

2. P. ANGELI BERNARDINI, « Esaltazione e critica dell'atletismo nella poesia greca dal VII al V sec. a.C. Storia di un ideologia", Stadion 6 (1980), p.81-111. L'A. a également produit une édition commentée de l'Anacharsis : Luciano: Anacarsi o sull'atletica, Pordenone, 1995.

3. Pour une synthèse de l'état de la recherche et des questions débattues, voir D. KAGAN, G.F. VIGGIANo (ed.), Men of Bronze: Hoplite Warfare in Ancient Greece, Princeton, 2013.

4. Exemples typiques de la première tendance : St. MÜLLER, «'Herrlicher Ruhm im Sport oder im Krieg' - Der Apobates und die Funktion des Sport in der griechischen Polis », Nikephoros 9 (1996), p. 41-69 ; Ch. MANN, Athlet und Polis im archaischen und frühklassischen Griechenland, Göttingen, 2001. L'autre approche a été illustrée récemment par D.M. PRITCHARD, Sport, Democracy and War in Classical Athens, Cambridge 2013. Gymnases et armées thébaines au IV siècle : cf. Diod. XV, 50, 5.

5. Par exemple, l'anecdote sur Alcée provient d'Hérodote V, 95 (et non 94 - p. 121) ; l'éphébie est décrite au chapitre 42 (et non 40) de la Constitution des Athéniens (p. 190 n. 16); l'allusion à M. Golden, Sport and Society in Ancient Greece, renvoie sans doute à la p. 25 et non 205 (p. 184); quant à l'inscription de Phaÿllos, on préfèrera citer l'édition IG I ${ }^{3} 823$ plutôt qu'IG I 655 (p. 69).

\section{AUTEURS}

\section{VINCENT CUCHE}

Université de Nice - UMR 7264 CEPAM 